\title{
Electrically Conductive Electrospun Polymeric Mats for Sensing Dispersed Vegetable Oil Impurities in Wastewater
}

\author{
Abdolali Moghaddasi ${ }^{1} @$, Patrik Sobolčiak ${ }^{2}$, Anton Popelka ${ }^{2}$, Kishor Kumar Sadasivuni ${ }^{2}$, \\ Zdeno Spitalsky ${ }^{3}\left(\mathbb{D}\right.$ and Igor Krupa ${ }^{2, *}$ \\ 1 College of College of Arts and Sciences, Qatar University, P.O. Box 2713 Doha, Qatar; amoghadasi@qu.edu.qa \\ 2 Center for Advanced Materials, Qatar University, P.O. Box 2713 Doha, Qatar; patrik@qu.edu.qa (P.S.); \\ anton.popelka@qu.edu.qa (A.P.); Kishorkumars@qu.edu.qa (K.K.S.) \\ 3 Polymer Institute Slovak Academy of Sciences, Dubravska Cesta 9, 84541 Bratislava, Slovakia; \\ zdeno.spitalsky@savba.sk \\ * Correspondence: igor.krupa@qu.edu.qa
}

Received: 10 November 2019; Accepted: 28 November 2019; Published: 2 December 2019

check for updates

\begin{abstract}
This paper addresses the preparation of electrically conductive electrospun mats on a base of styrene-isoprene-styrene copolymer (SIS) and multiwall carbon nanotubes (CNTs) and their application as active sensing elements for the detection of vegetable oil impurities dispersed within water. The most uniform mats without beads were prepared using tetrahydrofuran (THF)/ dimethyl formamide (DMF) 80:20 (v/v) as the solvent and $13 \mathrm{wt}$ \% of SIS. The CNT content was $10 \mathrm{wt} . \%$, which had the most pronounced changes in electrical resistivity upon sorption of the oil component. The sensors were prepared by deposition of the SIS/CNT layer onto gold electrodes through electrospinning and applied for sensing of oil dispersed in water for 50, 100, and $1000 \mathrm{ppm}$.
\end{abstract}

Keywords: nanocomposites; sensors; carbon nanotubes

\section{Introduction}

Continuous improvement of people's living standards is associated with intensive catering industry development, which results in a large amount of restaurant wastewater contaminated by various amounts of vegetable and animal oils. Untreated direct discharge of catering wastewater can reach more than 100 million tons each year [1]; however, untreated restaurant wastewater is not allowed to be discharged directly into the municipal sewerage network [1]. Domestic wastewater commonly contains average oil and grease contents in the range from 50 to $150 \mathrm{mg} / \mathrm{L}$; however, local contents may be significantly higher (over $400 \mathrm{ppm}$ ) [2]. Several international conventions regulate the concentration of oil, which can be discharged to the environment from 15 to $42 \mathrm{ppm}$ [3].There are several analytical techniques, such as measuring total organic content, spectroscopy, and chromatography, to measure contamination of water by oil. However, these techniques require using costly and non-compact instruments. Hence, a cheap, simple, and portable tool to detect oil contamination would be useful.

One approach to detect oil contamination is based on using sensors for liquids and gases based on electrically conductive polymer composites (ECPCs) designed from various kinds of polymeric matrices and electrically conductive fillers [4]. The principle of sensing is that the ECPC resistivity increases once the material is exposed to organic solvent vapor or liquid due to wetting of the ECPC by the organic solvent and diffusion into the composite. This results in swelling of the polymer matrix, thereby increasing the space between conductive fillers, which increases the resistivity. In addition to the solubility of the solvent in the polymer, the sensing capability depends on the thickness of the sensing 
element, the content of the conductive filler, and the organic vapor saturated pressure [5]. Typically, for ECPC preparation, either the solution or hot melting mixing is used to incorporate conductive nanofillers into the polymer matrix. This is followed by casing, hot melt pressing, or electrospinning [6]. The distribution, size, and geometrical parameters (e.g., aspect ratio) of the nanoparticles are crucial for reaching the percolation threshold, which is known as the critical volume content of conductive fillers at the transition of the polymer from being an insulator to being semi-conductive. For this reason, conductive nanofillers such as CNTs, graphite, and carbon black are often preferred because they can form a continuous conductive network with a matrix at low concentrations [7]. Among other applications, ECPCs have also found applicability in oil detection [8]. The principle is the selective absorption of oil components from a water/oil dispersion.

Herein, for the first time, the preparation of a simple, easy to use, and portable sensor for the detection of dispersed vegetable oil within water using electrically conductive nanocomposites based on styrene-isoprene-styrene copolymer and multiwall carbon nanotubes fabricated by electrospinning is reported. CNT fillers were used in this study in order to increase electrical conductivity of SIS electrospun mats. CNTs are relatively cheap, well-known fillers that are frequently used to reinforce various properties of polymeric composites.

In order to prepare the active layer of a sensor, the electrospinning approach possess several advantages such as an ability to tailor the porosity of the layers and the size of individual electrospun fibers. This opens the possibility of easily optimizing swelling characteristics of the sensor.

\section{Materials and Methods}

\subsection{Materials}

The following materials were used in this study: Styrene-isoprene-styrene (SIS) tri-block copolymer, $30 \mathrm{wt} . \%$ polystyrene (KRATON D1165 PT, KRATON POLYMERS, Belpre, OH, USA), multiwall carbon nanotubes (CNTs) with an outer diameter of 20-30 nm and a length of 10-30 $\mu \mathrm{m}$ (cheaptubes.com), dimethyl formamide (DMF) (Sigma Aldrich, St. Louis, MO, USA), tetrahydrofuran (THF) (Sigma Aldrich, USA), and sunflower vegetable oil (Adams Group, Arbuckle, CA, USA).

\subsection{Preparation of Solutions for Electrospinning}

SIS was diluted in a mixed solvent of THF and DMF 80:20 (v/v) at room temperature for $20 \mathrm{~h}$ and subsequently used for electrospinning.

In the case of a solution of SIS and CNTs, CNTs were first added to a DMF/THF solution followed by two drops of Triton $X$, and were then sonicated using an internal probe for $30 \mathrm{~min}$. Then, SIS was added to a mixture of CNTs and solvent and stirred for $5 \mathrm{~h}$ at room temperature. This procedure led to the formation of a homogeneous, long-term stable dispersion. Based on the optimization protocol, the SIS content in the solution was $13 \mathrm{wt} . \%$ and the concentration of CNTs was $10 \mathrm{wt} . \%$ relative to the polymer concentration.

\subsection{Electrospinning}

Electrospun nanofibers were fabricated using a NaBond (Shenzhen, China) electrospinning device. Typically, $5 \mathrm{~mL}$ of SIS/CNT dispersion was added into a $10 \mathrm{~mL}$ syringe with a blunt-end, stainless steel needle type 8 . The needle was connected to the emitting electrode of a high voltage supply capable of generating DC voltages. Aluminum foil was used as the collection screen, which connected to the ground electrode of the power supply. The distance between the screen and the needle tip was $15 \mathrm{~cm}$. The electrospinning process was carried out at room temperature at a voltage of $15 \mathrm{kV}$ for $20 \mathrm{~min}$ at a flow rate of $1.0 \mathrm{~mL} / \mathrm{h}$. 


\subsection{Fabrication of the Sensor}

Gold interdigitated electrodes were used for fabrication of the sensors as shown in Figure 1. The electrodes were placed onto aluminum foil in an electrospinning device. The electrospinning process was performed under the conditions described in part 2.3 using solutions composed of $13 \mathrm{wt} . \%$ SIS and $10 \mathrm{wt} \% \mathrm{CNT}$ for $20 \mathrm{~min}$ under $20 \mathrm{kV}$ and a flow rate of $2 \mathrm{~mL} / \mathrm{h}$. The samples were dried in the oven for $24 \mathrm{~h}$ at $60{ }^{\circ} \mathrm{C}$. The average weight of the electrospun layer was $10 \mathrm{mg} \pm 2 \mathrm{mg}$, and the average thickness was approximately $100 \pm 10 \mu \mathrm{m}$.

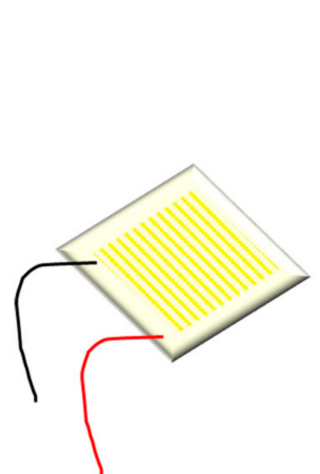

Gold electrode

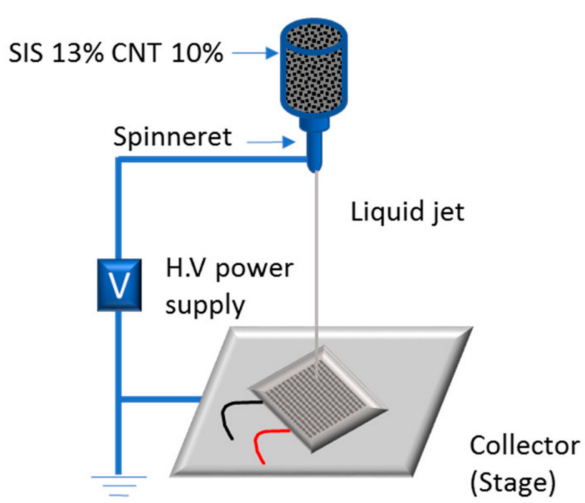

Electrospinning
SIS $13 \%$ CNT $10 \%$ E.S mat

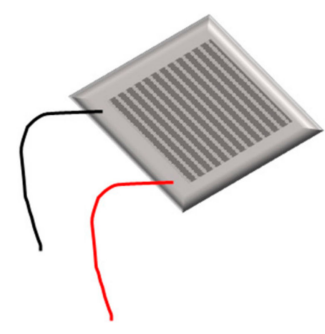

Sensor

Figure 1. Sketch of a sensor fabrication by electrospinning, with $13 \mathrm{wt} . \%$ of styrene-isoprene-styrene (SIS) copolymer and $10 \mathrm{wt} . \%$ of multiwall carbon nanotubes (CNTs) on a gold electrode.

\subsection{Oil Sensing Measurements}

Water, vegetable oil, and their mixtures with concentrations of 100 and 1000 ppm of vegetable oil were prepared by mixing water and vegetable oil with internal probe sonication for $20 \mathrm{~min}$ and then stirring with a magnetic stirrer overnight. A drop of emulsion was applied onto a prepared sensor to ensure full coverage of the active area of the sensor, and resistivity was subsequently measured.

Resistivity changes were measured by using the KEITHLEY 2635A system source meter upon exposure to drops of different oil mixtures over time.

Terminal-coated electrodes were connected to a source meter system (KEITHLEY 2635A, Austin, TX, USA) under $10 \mathrm{~mA}$ to measure the resistivity of the samples. The relative resistivity ( $\mathrm{A}_{\mathrm{R}}$ ) for each sensor was calculated using Equation (1):

$$
\mathrm{A}_{\mathrm{R}}=\left(\mathrm{R}-\mathrm{R}_{0}\right) / \mathrm{R}_{0} \times 100 \%,
$$

where $R$ is the resistance at time $t$ and $R_{0}$ is the initial resistance of the specimen. Each measurement was repeated five times.

\section{Results and Discussion}

\subsection{Morphology of the Electrospun Mats}

To prepare bead-less fibers with uniform thickness, different DMF/THF compositions, polymer concentration, and electrospinning conditions were tested. The best ratio of THF/DMF solvent was found to be $80 / 20(v / v)$, which is in agreement with a previous study [9]. In terms of concentration, the most uniform fibers were obtained using $13 \mathrm{wt}$ \% SIS (Figure 2a). The most suitable mat for further sensor preparation was composed of a solution of $13 \mathrm{wt}$ \% of SIS and $10 \mathrm{wt} . \%$ CNT and displayed a uniform fiber size with no defects or voids in the structure, as shown in Figure $2 b$. 

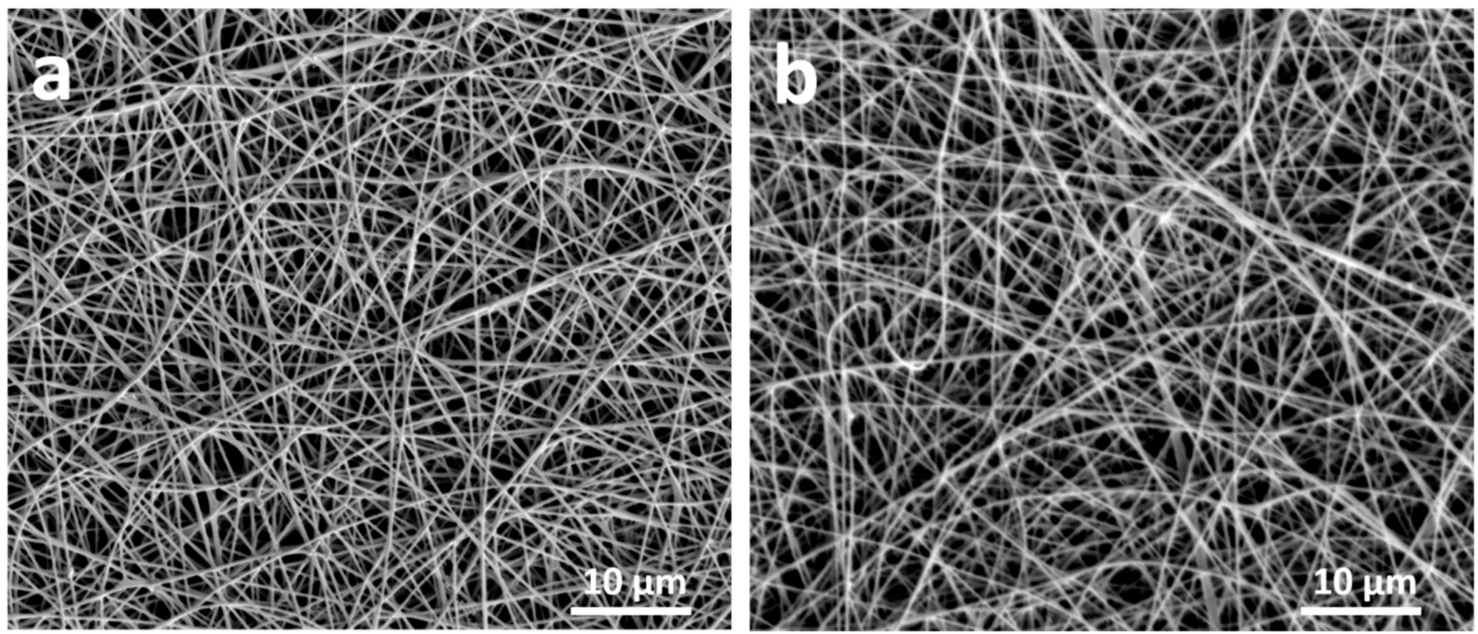

Figure 2. SEM image of (a) 13 wt.\% of SIS and (b) 13 wt.\% of SIS and $10 \mathrm{wt} . \%$ of CNT in tetrahydrofuran (THF)/dimethyl formamide (DMF) 80:20 (v/v).

\subsection{Sorption Capacity Determination}

To estimate the sorption capacity of SIS (electrospun mat and casted film) by oil, square pieces $(2 \times 2 \mathrm{~cm} ; 100 \mu \mathrm{m}$ thickness) of electrospun mat and casted film were immersed into vegetable oil for one hour and $24 \mathrm{~h}$. Subsequently, the sorption capacity was determined from the mass changes of the samples. It was found that electrospun SIS absorbed $3.31 \mathrm{~g}$ of oil per $1 \mathrm{~g}$ of SIS within the first hour with no further absorption. Casted SIS film of the same dimensions and geometry absorbed $0.35 \mathrm{~g}$ of oil per $1 \mathrm{~g}$ of SIS after $1 \mathrm{~h}$ of immersion and $3.34 \mathrm{~g}$ of oil per $1 \mathrm{~g}$ of film after $20 \mathrm{~h}$ of immersion in the oil. This simple test indicates significantly faster sorption of oil into the electrospun mat than the casted film. However, the equilibrium state was the same for both systems. The same test was performed for water, and it was found that both samples absorbed less than $0.5 \mathrm{wt} . \%$ of water.

\subsection{Oil Detection in Water/Oil Emulsions}

The changes in the relative resistivity of the electrospun mats poured into distilled water and vegetable oil are shown in Figure 3a. As expected, water did not influence the $A_{R}$ of the material. On the other hand, the sensor $A_{R}$ increased dramatically after pouring the sensor into oil, and after $3 \mathrm{~min}$, the value reached a plateau.

Mixtures of vegetable oil within water (50,100, and $1000 \mathrm{ppm}$ ) were used to test the sensitivity of the sensors for oil content detection.

Figure $3 \mathrm{~b}$ shows the $A_{R}$ values of $13 \mathrm{wt} . \%$ SIS and $10 \mathrm{wt} . \% \mathrm{CNT}$ for 50,100 , and $1000 \mathrm{ppm}$ vegetable oil in water. The $A_{R}$ of the SIS-CNT sensor increased after applying the oil mixture. A steep increase in the $A_{R}$ was observed after $7 \mathrm{~min}$ for $50 \mathrm{ppm}$ of oil in water. Similarly, an increase of $A_{R}$ after $5 \mathrm{~min}$ appeared for $100 \mathrm{ppm}$ of oil in water and after $4 \mathrm{~min}$ for $1000 \mathrm{ppm}$ oil in water. The fastest response for the 1000 ppm mixture was caused by the higher concentration gradient of oil, which caused the SIS fibers to swell at a faster rate, leading to an increase in the distance between CNTs and an increase in resistivity. The detection of oil impurities below $50 \mathrm{ppm}$ was complicated and ambiguous due to low changes in the electrical resistivity. 

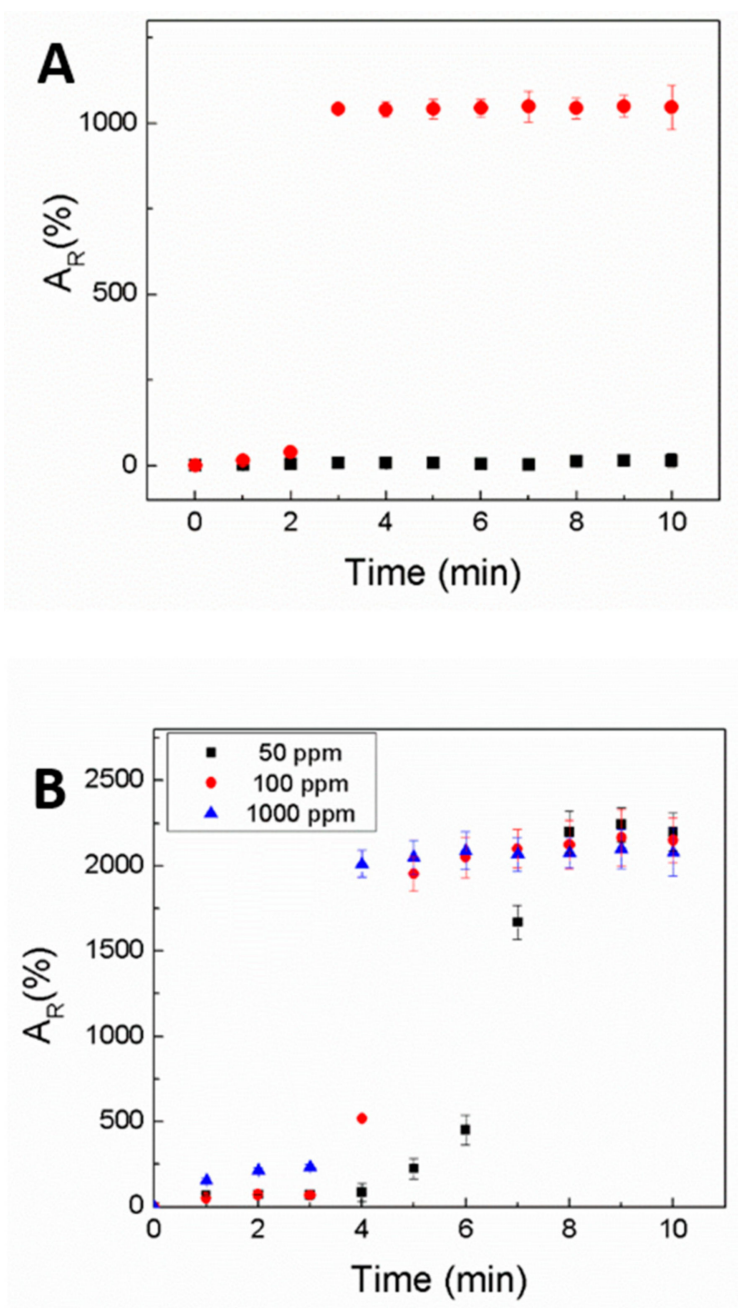

Figure 3. Relative resistivity of the SIS/CNT sensor for (A) water (black squares) and pure vegetable oil (red circles), and (B) 50 ppm (black squares), 100 ppm (red circles), and 1000 ppm (blue triangles) of vegetable oil.

\section{Conclusions}

The preparation of electrospun mats from styrene-isoprene-styrene copolymer (SIS) and multiwall carbon nanotubes was optimized. The most uniform mats without beads were prepared using THF/DMF 80:20 (v/v) as the solvent and $13 \mathrm{wt} . \%$ of SIS. The CNT content was $10 \mathrm{wt} . \%$ because that amount resulted in the most pronounced changes in electrical resistivity upon sorption of the oil component.

The sensors for detection of vegetable oil in water were designed by deposition of SIS/CNT mats onto gold electrodes through electrospinning.

Sensors were successfully applied for detection of oil dispersed in water in the range from 50 to $1000 \mathrm{ppm}$. The detection of oil impurities below $50 \mathrm{ppm}$ was complicated and ambiguous due to low changes in the electrical resistivity. Further research will be focused on the investigation of an influence of common water-soluble components occurring in waste water (e.g., salts) on oil detection ability. These impurities may interfere with the sensitivity of measurements; however, this is outside the scope of this paper.

Author Contributions: Conceptualization, I.K. and K.K.S.; methodology, I.K., P.S., and K.K.S.; validation, P.S., K.K.S., and I.K.; formal analysis, P.S., A.M., and A.P.; investigation, A.M., A.P., and P.S.; resources, I.K.; data curation, P.S., and A.M.; writing - original draft preparation, A.M., and P.S.; writing-review and editing, I.K., Z.S., and K.K.S.; supervision, Z.S., and I.K.; project administration, I.K.; funding acquisition, I.K. 
Funding: This publication was supported by the Qatar University Collaborative Grant QUCG-CAM-19/20-2. The findings achieved herein are solely the responsibility of the authors. The publication of this article was funded by the Qatar National Library.

Conflicts of Interest: The authors declare no conflict of interest.

\section{References}

1. Li, W.; Zhang, L.; Mu, D.; Pei, Y.; Ma, Y.; Ma, M.; Dou, W. Treatment of restaurant waste water with $\mathrm{Al}_{2} \mathrm{O}_{3}$ ceramic membrane. Am. J. Water Sci. Eng. 2018, 4, 28-32. [CrossRef]

2. Tchobanoglous, G.; Burton, F.L.; Franklin, L.; Stensel, H.D.; Metcalf \& Eddy. Wastewater Engineering: Treatment and Reuse; McGraw-Hill: New York, NY, USA, 2003; ISBN 0070418780.

3. Saththasivam, J.; Loganathan, K.; Sarp, S. An overview of oil-water separation using gas flotation systems. Chemosphere 2016, 144, 671-680. [CrossRef] [PubMed]

4. Liu, H.; Huang, W.; Yang, X.; Dai, K.; Zheng, G.; Liu, C.; Shen, C.; Yan, X.; Guo, J.; Guo, Z. Organic vapor sensing behaviors of conductive thermoplastic polyurethane-graphene nanocomposites. J. Mater. Chem. C 2016, 4, 4459-4469. [CrossRef]

5. Gao, J.-F.; Yan, D.-X.; Huang, H.-D.; Zeng, X.-B.; Zhang, W.-Q.; Li, Z.-M. Tunable positive liquid coefficient of an anisotropically conductive carbon nanotube-polymer composite. J. Polym. Res. 2011, 18, 2239-2243. [CrossRef]

6. Qiu, Y.; Wang, J.; Wu, D.; Wang, Z.; Zhang, M.; Yao, Y.; Wei, N. Thermoplastic polyester elastomer nanocomposites filled with graphene: Mechanical and viscoelastic properties. Compos. Sci. Technol. 2016, 132, 108-115. [CrossRef]

7. Pang, H.; Xu, L.; Yan, D.-X.; Li, Z.-M. Conductive polymer composites with segregated structures. Prog. Polym. Sci. 2014, 39, 1908-1933. [CrossRef]

8. Zheng, L.; Su, X.; Lai, X.; Chen, W.; Li, H.; Zeng, X. Conductive superhydrophobic cotton fabrics via layer-by-layer assembly of carbon nanotubes for oil-water separation and human motion detection. Mater. Lett. 2019, 253, 230-233. [CrossRef]

9. Feng, S.-Q.; Shen, X.-Y.; Fu, Z.-Y.; Ji, Y.-L. Studies on the electrospun submicron fibers of SIS and its mechanical properties. J. Appl. Polym. Sci. 2009, 114, 1580-1586. [CrossRef]

(C) 2019 by the authors. Licensee MDPI, Basel, Switzerland. This article is an open access article distributed under the terms and conditions of the Creative Commons Attribution (CC BY) license (http://creativecommons.org/licenses/by/4.0/). 